\title{
ON THE PRODUCT OF SEMI-GROUPS OF OPERATORS ${ }^{1}$
}

\author{
H. F. TROTTER
}

1. Introduction. We consider semi-groups of operators on a Banach space $X$, which are of class $\left(C_{0}\right)$ in the terminology of [3]. Such a semi-group is a family of bounded operators $T_{t}$, defined for all $t>0$ and satisfying the semi-group condition

$$
T_{t+s}=T_{t} T_{s}
$$$$
s, t>0
$$

and the continuity condition

$$
\lim _{t \rightarrow 0} T_{t} f=f
$$$$
f \in X \text {. }
$$

The (infinitesimal) generator is the operator defined by

$$
\Omega=\lim _{t \rightarrow 0} t^{-1}\left(T_{t}-I\right) .
$$

Let $T_{t}^{\prime}$ be another semi-group of class $\left(C_{0}\right)$, with infinitesimal generator $\Omega^{\prime}$. If $T_{t}$ and $T_{s}^{\prime}$ commute for all values of $s$ and $t$, it is obvious that

$$
U_{a, t}=T_{t} T_{a t}^{\prime}
$$

is again a semi-group of class $\left(C_{0}\right)$, for any fixed positive $a$. If the commutativity does not hold, we may still attempt to define "product" semi-groups $S_{a, t}$ by

$$
S_{a, t}=\lim _{h \rightarrow 0}\left(T_{h} T_{a h}^{\prime}\right)^{[t / h]}
$$

where $[t / h]$ is the greatest integer in $t / h$. The symbolic equation $T_{t}=e^{\Omega t}$ suggests that $U_{a, t}$ and $S_{a, t}$ should have $\Omega+a \Omega^{\prime}$ as infinitesimal generator. The situation is fairly simple in the commutative case, and is described in Theorem 1 below. From here on, it is to be understood that $T_{t}$ and $T_{t}^{\prime}$ are semi-groups of class $\left(C_{0}\right)$ with the respective generators $\Omega$ and $\Omega^{\prime}$.

THEOREM 1. If $T_{t}$ and $T_{s}^{\prime}$ commute for all values of $t$ and $s$, then for any positive $a$, the closure of $\Omega+a \Omega^{\prime}$ generates the semi-group $U_{a, t}$ defined by (4).

Presented to the Society, October 25, 1958; received by the editors November 3, 1958.

${ }^{1}$ Research done at Princeton University and supported in part by the Office of Ordnance Research, U. S. Army. 
Example 1 of $\S 3$ shows that $\Omega+a \Omega^{\prime}$ itself is not necessarily the generator.

In the noncommutative case we find it necessary to impose a condition on the norms of $T_{t}$ and $T_{t}^{\prime}$. It is a standard result [3, pp. $306,322]$ that conditions (1) and (2) imply the existence of constants $M, M^{\prime}, \omega, \omega^{\prime}$ such that

$$
\left\|T_{t}\right\| \leqq M e^{\omega t}, \quad\left\|T_{t}^{\prime}\right\| \leqq M^{\prime} e^{\omega^{\prime} t}
$$

for all $t>0$. We shall say that $T_{t}, T_{t}^{\prime}$ satisfy the norm condition if the constants $M, M^{\prime}$ may be taken equal to 1 .

If $A$ is an operator, we write $D(A)$ for the domain of $A$, (i.e., the set of $f$ for which $A f$ is defined) and $R(A)$ for the range of $A$ (i.e., the set of $g$ such that $g=A f$ for some $f$ ).

Lemma. Suppose that $T_{t}$ and $T_{t}^{\prime}$ satisfy the norm condition and that $D\left(\Omega+a \Omega^{\prime}\right)=D(\Omega) \cap D\left(\Omega^{\prime}\right)$ is dense in $X$. Then [the closure of] $\Omega+a \Omega^{\prime}$ generates a semi-group of class $\left(C_{0}\right)$ if and only if $R\left(\lambda-\Omega-a \Omega^{\prime}\right)$ is [dense in] $X$ for some $\lambda>\omega+a \omega^{\prime}$. If $\Omega+a \Omega^{\prime}$ (or its closure) generates a semi-group of class $\left(C_{0}\right)$, the generated semi-group is given by (5).

REMARK. If the closure of $\Omega+a \Omega^{\prime}$ is the infinitesimal generator of a semi-group of class $(A)$ (for the notation, see [3]) then $R\left(\lambda-\Omega-a \Omega^{\prime}\right.$ ) is dense in $X$ for all sufficiently large $\lambda[3$, p. 344]. Hence, under the hypotheses of the lemma, if $\Omega+a \Omega^{\prime}$, or its closure, generates a semigroup of class $(A)$, then it actually generates one of class $\left(C_{0}\right)$ which is given by (5).

Theorem 2. Suppose that $T_{t}$ and $T_{t}^{\prime}$ satisfy the norm condition, and that $D(\Omega) \subset D\left(\Omega^{\prime}\right)$. Then the set of numbers a such that $\Omega+a \Omega^{\prime}$ generates a semi-group of class $\left(C_{0}\right)$ is open in $[0, \infty)$ and contains a neighbourhood of 0 .

It is an immediate consequence that under the hypotheses of Theorem 2 the set of numbers $a$ such that the closure of $\Omega+a \Omega^{\prime}$ generates a semi-group of class $\left(C_{0}\right)$ contains a neighbourhood of the origin. Example 3 of $\$ 3$ shows that in a sense this is the most that can be said.

Theorems 1 and 2 may be regarded as perturbation theorems in the sense of Phillips, since they assert that under certain conditions, when an infinitesimal generator $\Omega$ is modified by adding another operator $a \Omega^{\prime}$, the modified operator (or its closure) is again an infinitesimal generator. The results are rather different from those of Phillips [3], in that the set of perturbing operators is not linear, nor even (in the noncommutative case) a positive cone. In $\$ 3$ we exhibit 
two groups of class $\left(C_{0}\right)$ which satisfy the norm condition and have infinitesimal generators $\Omega, \Omega^{\prime}$ with the same domain, such that the closure of $\Omega+\Omega^{\prime}$ does not generate a semi-group of class $(A)$. In $\S 4$ we discuss the norm condition and illustrate a type of misbehaviour which may occur if it is not satisfied. The example used here also shows the existence of two groups of class $\left(C_{0}\right)$ whose infinitesimal generators have the same domain, such that no extension of $\Omega+\Omega^{\prime}$ generates a semi-group of class $(A)$. This improves on an example of Dye and Phillips [1] and answers a question raised in [3, p. 417].

2. Proofs. We first remark that $h^{-1}\left(T_{h} T_{a h}^{\prime}-I\right)$ may be written $h^{-1}\left(T_{h}-I\right)+a(a \dot{h})^{-1} T_{h}\left(T_{a h}^{\prime}-I\right)$, which shows that

$$
\Omega_{a}=\lim _{h \rightarrow 0} h^{-1}\left(T_{h} T_{a h}^{\prime}-I\right)
$$

is an extension of $\Omega+a \Omega^{\prime}$. It follows that if $D\left(\Omega+a \Omega^{\prime}\right)$ and $R\left(\lambda-\Omega-a \Omega^{\prime}\right)$ are dense in $X$, then so are $D\left(\Omega_{a}\right)$ and $R\left(\lambda-\Omega_{a}\right)$.

Under the hypotheses of Theorem 1 , it is obvious that the operators $U_{a, t}$ defined by (4) constitute a semi-group of class $\left(C_{0}\right)$ and that $\Omega_{a}$ is its infinitesimal generator. Hence $\Omega_{a}$ is a closed extension of $\Omega+a \Omega^{\prime}$, and we need only show that it is the minimal closed extension. For $\lambda$ sufficiently large, $J_{\lambda}=(\lambda-\Omega)^{-1}$ and $J_{\lambda}^{\prime}=\left(\lambda-\Omega^{\prime}\right)^{-1}$ are bounded operators and

$$
\lim _{\lambda \rightarrow \infty} \lambda J_{\lambda}=\lim _{\lambda \rightarrow \infty} \lambda J_{\lambda}^{\prime}=I .
$$

Also $R\left(J_{\lambda}\right)=D(\Omega)$, and for $f \in D(\Omega), J_{\lambda} \Omega f=\Omega J_{\lambda} f$ [3, Chapter XI]. From the fact that $J_{\lambda}=\int_{0}^{\infty} e^{-\lambda t} T_{t} d t$ and $J_{\lambda}^{\prime}=\int_{0}^{\infty} e^{-\lambda t} T_{t}^{\prime} d t$ it is clear that the commutativity of $T_{t}$ and $T_{s}^{\prime}$ implies that $J_{\lambda} J_{\lambda}^{\prime}=J_{\lambda}^{\prime} J_{\lambda}$. Letting $K_{\lambda}=\lambda^{2} J_{\lambda} J_{\lambda}^{\prime}=\lambda^{2} J_{\lambda}^{\prime} J_{\lambda}$ we see that $\lim _{\lambda \rightarrow \infty} K_{\lambda}=I$ and that $R\left(K_{\lambda}\right) \subset R\left(J_{\lambda}\right) \cap R\left(J_{\lambda}^{\prime}\right)=D(\Omega) \cap D\left(\Omega^{\prime}\right)=D\left(\Omega+a \Omega^{\prime}\right)$. Since $K_{\lambda}$ commutes with $U_{a, t}$, it also commutes with $\Omega_{a}$ in the sense that if $f \in D\left(\Omega_{a}\right)$ then $\Omega_{a} K_{\lambda} f=K_{\lambda} \Omega_{a} f$. Since $\Omega_{a}$ is an extension of $\Omega+a \Omega^{\prime}$ and $R\left(K_{\lambda}\right) \subset D\left(\Omega+a \Omega^{\prime}\right)$, we have $K_{\lambda} \Omega_{a} f=\left(\Omega+a \Omega^{\prime}\right) K_{\lambda} f$ for any $f \in D\left(\Omega_{a}\right)$. As $\lambda \rightarrow \infty, K_{\lambda} f \rightarrow f$ and $\left(\Omega+a \Omega^{\prime}\right) K_{\lambda} f=K_{\lambda} \Omega_{a} f \rightarrow \Omega_{a} f$. Hence, every closed extension of $\Omega+a \Omega^{\prime}$ is an extension of $\Omega_{a}$, or in other words, $\Omega_{a}$ is the closure of $\Omega+a \Omega^{\prime}$. This completes the proof of Theorem 1 .

If $T_{t}$ and $T_{t}^{\prime}$ both satisfy the norm condition then

$$
\left\|\left(T_{h} T_{a h}^{\prime}\right)^{k}\right\| \leqq e^{\left(\omega+a \omega^{\prime}\right) k h}
$$

for all $h, k$. According to Theorem 5.3 of [4], (8) and the remark following (7) imply that if $D\left(\Omega+a \Omega^{\prime}\right)$ and $R\left(\lambda-\Omega-a \Omega^{\prime}\right)$ are dense in $X$ for some $\lambda>\omega+a \omega^{\prime}$, then $\bar{\Omega}_{a}$, the closure of $\Omega_{a}$, generates a semi-group 
of class $\left(C_{0}\right)$ which is given by (5). For $\lambda>\omega+a \omega^{\prime}, \lambda-\bar{\Omega}_{a}$ has a bounded inverse, and hence $\left(\lambda-\Omega-a \Omega^{\prime}\right)^{-1}\left(\right.$ defined on $R\left(\lambda-\Omega-a \Omega^{\prime}\right)$ ) exists and is bounded. If $R\left(\lambda-\Omega-a \Omega^{\prime}\right)$ is dense in $X$, the closure of $\left(\lambda-\Omega-a \Omega^{\prime}\right)^{-1}$ must be $J_{a, \lambda}$ which shows that $\bar{\Omega}_{a}$ is the closure of $\Omega+a \Omega^{\prime}$. Similarly, if $R\left(\lambda-\Omega-a \Omega^{\prime}\right)=X$ we have $\bar{\Omega}_{a}=\Omega+a \Omega^{\prime}$. The converse propositions are trivial, and this completes the proof of the lemma.

Under the hypotheses of Theorem $2, D\left(\Omega+a \Omega^{\prime}\right)=D(\Omega)$ and is consequently dense in $X$. Hence, according to the lemma, $\Omega+a \Omega^{\prime}$ generates a semi-group if and only if $R\left(\lambda-\Omega-a \Omega^{\prime}\right)=X$ for some $\lambda>\omega+a \omega^{\prime}$. We also have, for sufficiently large $\lambda, R\left(J_{\lambda}\right)=D(\Omega)$ $\subset D\left(\Omega^{\prime}\right)$, so that $\Omega^{\prime} J_{\lambda}$ is everywhere defined. Since $\Omega^{\prime}$ is closed and $J_{\lambda}$ is bounded, $P_{\lambda}=\Omega^{\prime} J_{\lambda}$ is closed and hence bounded. Since $D\left(\Omega+a \Omega^{\prime}\right)$ $=D(\Omega)=R\left(J_{\lambda}\right), \quad R\left(\lambda-\Omega-a \Omega^{\prime}\right)=R\left(\left(\lambda-\Omega-a \Omega^{\prime}\right) J_{\lambda}\right)=R\left(I-a P_{\lambda}\right)$ $=R\left(a^{-1}-P_{\lambda}\right)$. Therefore if $a^{-1}$ is in $\rho\left(P_{\lambda}\right)$, the resolvent set of $P_{\lambda}$, $R\left(\lambda-\Omega-a \Omega^{\prime}\right)=X$. Conversely, if $\Omega+a \Omega^{\prime}$ generates a semi-group, $\left(\lambda-\Omega-a \Omega^{\prime}\right)=a\left(a^{-1}-P_{\lambda}\right)(\lambda-\Omega)$ has an inverse defined on all of $X$ (provided $\lambda>\omega+a \omega^{\prime}$ ) and hence $a^{-1}$ is in the resolvent set of $P_{\lambda}$. Thus for $\omega+a \omega^{\prime}<\lambda, \Omega+a \Omega^{\prime}$ generates a semi-group if and only if $a^{-1}$ is in the resolvent set of $P_{\lambda}$. Consequently, $\Omega+a \Omega^{\prime}$ generates a semigroup if and only if $a^{-1}$ is in the union over all positive $\lambda$ of the sets $\left(\omega^{\prime}(\lambda-\omega)^{-1}, \infty\right) \cap \rho\left(P_{\lambda}\right)$. Since $P_{\lambda}$ is bounded, $\rho\left(P_{\lambda}\right)$ is open and contains a neighbourhood of infinity. Hence the set of values of $a$ for which $\Omega+a \Omega^{\prime}$ generates a semi-group is open and contains a neighbourhood of 0 .

3. Example 1. We take $X$ to be the Banach space of all continuous functions on the real line which vanish at infinity, with $\|f\|$ $=\max _{x}|f(x)|$. Let $T_{t}$ and $T_{t}^{\prime}$ be the semi-groups of left and right translations; i.e., $T_{t} f(x)=f(x-t)$ and $T_{t}^{\prime} f(x)=f(x+t)$. Then $\Omega=-D$, $\Omega^{\prime}=D$ where $D f$ is the derivative of $f$. (The domain of $D$ is the set of $f$ such that $f^{\prime} \in X$.) Clearly $\left\|T_{t}\right\|=\left\|T_{t}^{\prime}\right\|=1$ and the hypotheses of both Theorems 1 and 2 are satisfied. For $a \neq 1, \Omega+a \Omega^{\prime}$ does generate a semi-group, but $\Omega+\Omega^{\prime}$ is the zero operator restricted to $D(\Omega)$. Its closure generates the trivial semi-group, but the operator itself is not an infinitesimal generator.

The preceding example shows that it is more appropriate to expect the closure of $\Omega+\Omega^{\prime}$ to generate a semi-group. The next example shows that even this need not be true. (Note that in both Examples 1 and 2 we actually have groups of operators.)

Example 2. Let $X$ and $T_{\imath}$ be as in Example 1. Let $\phi$ be the continuous function defined by $\phi(x)=1$ for $x \leqq 0, \phi(x)=1+x^{1 / 2}$ for $0<x \leqq 1$, and $\phi(x)=2$ for $1<x$, and let $\psi(x)=\int_{0}^{x}[\phi(u)]^{-1} d u$. The func- 
tion $\psi$ is a homeomorphism of the real line onto itself, and we obtain a semi-group on $X$ by setting $T_{t}^{\prime} f(x)=f\left(\psi^{-1}(\psi(x)+t)\right)$. It is obvious that $\left\|T_{t}^{\prime}\right\|=1$, and it is easy to calculate that $\Omega^{\prime}=\phi(x) D$ so that $D(\Omega)=D\left(\Omega^{\prime}\right)$. We shall show that $S_{t}=\lim _{h \rightarrow 0}\left(T_{h} T_{h}^{\prime}\right)^{[t / h]}$ is not everywhere defined, so that according to the remark following the statement of the lemma in $\S 1$, the closure of $\Omega+\Omega^{\prime}$ cannot generate a semigroup of type $(A)$. For $x<-h, T_{h} T_{h}^{\prime} f(x)=f(x)$, so that if $S_{t} f$ is defined, $S_{t} f(x)=f(x)$ for all $x<0$. Now take $k>0$ and define $\bar{\phi}(x)$ $=\phi(x)$ for $x<k, \bar{\phi}(x)=\phi(k)$ for $x \geqq k$. Let $\bar{\psi}(x)=\int_{0}^{x}[\bar{\phi}(u)]^{-1} d u$ and define $\bar{T}_{t}$ by $\bar{T}_{t} f(x)=f\left(\bar{\psi}^{-1}(\bar{\psi}(x)+t)\right)$. It is easily seen that for $x>k$, $\left(T_{h} T_{h}^{\prime}\right)^{n} f(x)=\left(T_{h} \bar{T}_{h}\right)^{n} f(x)$. Now $\Omega+\bar{\Omega}$ (where $\bar{\Omega}$ generates $\bar{T}_{t}$ ) is equal to $\chi(x) D$ where $\chi(x)=k^{1 / 2}$ for $x \leqq k, \chi(x)=x^{1 / 2}$ for $k<x \leqq 1$ and $\chi(x)=1$ for $1<x$. Defining $\xi(x)=\int_{0}^{x}[\chi(u)]^{-1} d u$, it is easy to check that $\Omega+\bar{\Omega}$ generates $\bar{S}_{t}$ with $\bar{S}_{t} f(x)=f\left(\xi^{-1}(\xi(x)+t)\right)$. Applying the lemma and the remarks above, we obtain the result that if $S_{t} f$ is defined

$$
\begin{aligned}
S_{t} f(x) & =\lim _{h \rightarrow 0}\left(T_{h} T_{h}^{\prime}\right)^{[t / h]} f(x) \\
& =\lim _{h \rightarrow 0}\left(T_{h} \bar{T}_{h}\right)^{[t / h]} f(x) \\
& =\bar{S}_{t} f(x)=f\left(\left(2 x^{1 / 2}+t\right)^{2} / 2\right)
\end{aligned}
$$

provided $x>k$ and $2 x^{1 / 2}+t \leqq 1$. Since $k$ was an arbitrary positive number (9) holds for all sufficiently small positive $x$. Now $S_{t} f$ is a continuous function (if it exists), so we have $f(0)=\lim _{x \uparrow 0} S_{t} f(x)$ $=\lim _{x \downarrow 0} S_{t} f(x)=f\left(t^{2} / 2\right)$, provided $t<1$. (For $t \geqq 1$ we actually have $\lim _{x \downarrow 0} S_{t} f(x)=f(t-1 / 2)$.) Hence $S_{t} f$ is not defined for all $f$.

If $a \in(0,1 / 2)$ or $(1, \infty)$ it is clear that $\Omega+a \Omega^{\prime}$ generates a semigroup similar to those described above, while for $a \in[1 / 2,1]$, $\Omega+a \Omega^{\prime}$ is not closed and hence is not an infinitesimal generator. It is perhaps less obvious that for $a \in[1 / 2,1)$, the closure of $\Omega+a \Omega^{\prime}$ is a generator. We shall describe briefly the semi-groups generated, leaving the verification of details to the reader. For $a \in(1 / 2,1), \Omega+a \Omega^{\prime}$ has the form $\eta(x) D$ where $\eta$ vanishes at some point $k \in(0,1)$. One obtains homeomorphisms $\psi_{1}$ and $\psi_{2}$ of $(-\infty, k)$ and $(k, \infty)$ onto $(-\infty, \infty)$ by defining $\psi_{1}(x)=\int_{0}^{x}[\eta(u)]^{-1} d u$ and $\psi_{2}(x)=\int_{1}^{x}[\eta(u)]^{-1} d u$. $S_{a, t}$ is then given by $\left.S_{a, t} f(k)=k, S_{a, t} f(x)=\psi_{1}^{-1}\left(\psi_{1}(x)+t\right)\right)$ for $x<k$, and $\left.S_{a, t} f(x)=\psi_{2}^{-1}\left(\psi_{2}(x)+t\right)\right)$ for $x>k$. For $a=1 / 2, S_{a, t} f(x)$ is defined similarly for $x<1$, while $S_{a, t} f(x)=f(x)$ for $x \geqq 1$.

In this example the closure of $\Omega+a \Omega^{\prime}$ failed to be a generator only for $a=1$. By using $T_{\alpha t}$ in place of $T_{t}$, the failure could be made to occur for $a=\alpha$. We next construct an example in which the set of $a$ 's for which failure occurs is an arbitrary subset of $(0, \infty)$. 
ExAmple 3. Let $A$ be any nonempty subset of $(0, \infty)$. For each $\alpha \in A$, take a Banach space $X_{\alpha}$ and two semi-groups $T_{\alpha, t}, T_{\alpha, t}^{\prime}$ having norm 1 and $D\left(\Omega_{\alpha}\right)=D\left(\Omega_{\alpha}^{\prime}\right)$. Suppose that the closure of $\Omega_{\alpha}+a \Omega_{\alpha}^{\prime}$ generates a semi-group for $a \neq \alpha$, while $\lim _{h \rightarrow 0}\left(T_{\alpha, h} T_{\alpha, \alpha h}^{\prime}\right)^{[t / h]}$ fails to be everywhere defined. We shall also suppose that for $f \in D\left(\Omega_{\alpha}\right)$

$$
\left\|\Omega_{\alpha} f\right\| \leqq \alpha\left\|\Omega_{\alpha}^{\prime} f\right\| \leqq 2\left\|\Omega_{\alpha} f\right\| .
$$

Semi-groups with these properties are easily constructed by the method of Example 2. Now let $X$ be the " $l_{1}$-space" formed from the $X_{\alpha}$; i.e., the elements of $X$ are functions $f$ defined on $A$ such that $f_{\alpha} \in X_{\alpha}$ and $\|f\|=\sum_{\alpha \in A}\left\|f_{\alpha}\right\|$ is finite. (This implies that $f_{\alpha}=0$ for all but a countable number of $\alpha$.) Under the norm defined above, $X$ is a Banach space and $X_{F}$, the set of $f$ such that $f_{\alpha}=0$ except for a finite number of $\alpha$, is a dense subset. The semi-groups defined on each $X$ induce semi-groups $T_{t}, T_{t}^{\prime}$ on $X$ which have norm 1 and are of class $\left(C_{0}\right)$. For $a \in A, \lim _{h \rightarrow 0}\left(T_{h} T_{a h}^{\prime}\right)^{[t / h]}$ is not everywhere defined, so that the closure of $\Omega+a \Omega^{\prime}$ cannot be a generator. On the other hand, for $a \notin A, D\left(\Omega+a \Omega^{\prime}\right)$ and $R\left(\lambda-\Omega-a \Omega^{\prime}\right)$ are dense in $X_{F}$ and hence in $X$, so that the lemma implies that the closure of $\Omega+a \Omega^{\prime}$ does generate a semi-group. Under condition (10), $D(\Omega) \subset D\left(\Omega^{\prime}\right)$ if and only if $A$ is bounded from zero, which is in agreement with Theorem 2 .

4. The norm condition. In the proof of the lemma, the hypothesis that $T_{t}$ and $T_{t}^{\prime}$ satisfy the norm condition is used only to obtain (8). If (8) were weakened by replacing the right-hand side by $M e^{\left(\omega+a \omega^{\prime}\right) k h}$, Theorem 5.3 of [4] could still be applied to give the desired result. This suggests that the norm condition may be superfluous. The following example, in which the weakened form of (8) does not hold makes the suggestion less plausible.

EXAmple 4 . Let $X$ be $L^{1}(-\infty, \infty)$, and define $T_{t}$ by $T_{t} f(x)$ $=f(x+t)$ and $T_{t}^{\prime}$ by $T_{t}^{\prime} f(x)=\psi\left(\psi^{-1}(x)-t\right)$ where $\psi(x)=x$ for $x \leqq 0$, $\psi(x)=2 x$ for $x>0$. Then $\Omega=D, \Omega^{\prime}=\phi(x) D$ where $\phi(x)=-1$ for $x \leqq 0$, $\phi(x)=-2$ for $x>0$. (Here $D$ is again the operation of differentiation, but in this case its domain consists of the absolutely continuous functions in $L^{1}$ whose derivatives are in $L^{1}$.) We clearly have $D(\Omega)$ $=D\left(\Omega^{\prime}\right)$. For $h>0$, let $f_{h}$ be the characteristic function of the interval $[0, h]$. By direct calculation, $\left(T_{h} T_{h}^{\prime}\right)^{k} f_{h}$ is the characteristic function of $[0, k h]$. Since $\left\|f_{h}\right\|=h$ and $\left\|\left(T_{h} T_{h}^{\prime}\right)^{k} f_{h}\right\|=k h$, it follows that $\left\|\left(T_{h} T_{h}^{\prime}\right)^{k}\right\| \geqq k$ regardless of the value of $h$, so that $\left\|\left(T_{h} T_{h}^{\prime}\right)^{k}\right\|$ cannot be bounded by a function of the form $M e^{K k h}$.

Writing $\bar{\Omega}$ for the closure of $\Omega+\Omega^{\prime}$ it is easily shown that for any $\lambda>0$, the function $f$ defined by $f(x)=0$ for $x \leqq 0, f(x)=e^{-\lambda x}$ for $x>0$ 
is in $D(\bar{\Omega})$ and $(\lambda-\bar{\Omega}) f=0$. Thus for every $\lambda>0$, no closed extension of $\lambda-\left(\Omega+\Omega^{\prime}\right)$ can have an inverse, and this implies that no extension of $\Omega+\Omega^{\prime}$ generates a semi-group.

This last example has a further application. If $T_{t}$ is of class $\left(C_{0}\right)$ (and hence satisfies (1)), the space $X$ can be renormed with an equivalent norm (by defining $\|f\|^{\prime}$ as $\sup _{t>0} e^{-\omega t}\left\|T_{t} f\right\|$ ) so that $T_{t}$ satisfies the norm condition [2]. Example 4 shows that, given two semi-groups of class $\left(C_{0}\right)$, it is not necessarily possible to renorm the space so that both of them simultaneously satisfy the norm condition.

\section{REFERENCES}

1. H. A. Dye and R. S. Phillips, Groups of positive operators, Canad. J. Math. vol. 8 (1956) pp. 462-486.

2. W. Feller, On the generation of unbounded semi-groups of bounded linear operators, Ann. of Math. (2) vol. 58 (1953) pp. 166-174.

3. E. Hille and R. S. Phillips, Functional analysis and semi-groups, Amer. Math. Soc. Colloquium Publications, vol. 31, 1957.

4. H. F. Trotter, A pproximation of semi-groups of operators, Pacific J. Math. vol. 8 (1958) pp. 887-919.

QueEn's University, Kingston, Canada 